\title{
Insights About Induced SFR in the Shell Systems of Early-Type Galaxies from UV (GALEX)
}

\author{
L. M. Buson ${ }^{1}$, D. Bettoni ${ }^{1}$, A. Marino ${ }^{1}$, C. Chiosi ${ }^{2}$, G. Galletta ${ }^{2}$, \\ R. Rampazzo ${ }^{1}$, R. Tantalo ${ }^{2}$, and R. M. Rich \\ ${ }^{1}$ INAF - Osservatorio Astronomico di Padova \\ email:lucio.buson, daniela.bettoni, antonietta.marino, roberto.rampazzo@oapd.inaf.it \\ ${ }^{2}$ Dipartimento di Astronomia, Unversitá di Padova \\ email:giuseppe.galletta, cesare.chiosi, rosaria.tantalo@unipd.it \\ ${ }^{3}$ Physics \& Astron. Dept., UCLA email: rmr@astro.ucla.edu
}

\begin{abstract}
We present UV data of a small sample of shell galaxies. For the majority of them (namely, NGC 2865 and NGC 7135), the NUV matches the optical emission, while the FUV is present in the innermost regions alone.
\end{abstract}

Keywords. Galaxies, UV, Star Formation

\section{Results}

Shell galaxies represent the ideal class of objects to investigate galaxy evolution in the field for several reasons. In fact whatever their origin is, either a weak interaction (Thomson 1991) or a merging/accretion between galaxies (Dupraz and Combes (1986), Hernquist and Quinn (1987)), the event should have triggered a secondary burst of star formation. Here we present the images, obtained with Galaxy Evolution Explorer (GALEX) in far (FUV) and near (NUV) ultraviolet, of three nearby shell galaxies, namely NGC 2865, NGC 5018 and NGC 7135, whose optical line-strength indices (Longhetti et. al. 1999) suggest that they host recent ( $\leqslant 2$ Gyr) star formation. Their FUV emissionwhen available - is always confined in the innermost regions while the NUV emission shows fine structures very similar to those detected in the optical band. Remarkable in NGC 7135 is the coincidence between the optical and NUV fine structures which give to the galaxy a "medusa-like" morphology in both bands. NUV and FUV emissions likely have different origins (Dorman et al. 2003). The NUV emission in a passively evolving galaxy is dominated by the turnoff and sub-giant stars. The FUV emission traces one (or more) hot, plausibly high-metallicity, stellar components (e.g. hot-HB and/or post-AGB and AGB manquè stars) giving origin to their well-known UV-upturn phenomenon. At the same time, the UV light is also a sensitive tracer of the residual star formation in ETGs, a necessary ingredient to constrain the galaxy rejuvenation suggested by their optical line-strength indices which, however, suffer from age-metallicity degeneracy effects.

\section{References}

Thomson, R.C. 1991, MNRAS, 253, 256.

Dupraz, C., Combes, F. 1986, AAS, 166, 53.

Hernquist, L., Quinn, P. 1987, ApJ, 312, 1.

Longhetti, M., Bressan, A., Chiosi, C., Rampazzo, R. 1999. A\&A, 345, 419.

Dorman, B., Rood, R.T., \& O'Connell, R.W. 2003. ApJ, 591, 878. 